\title{
Applications of Deep Learning: A Review
}

\author{
Chen Ming ${ }^{1}$, Shen Jie ${ }^{2}$, N.Thirupathi $\mathrm{Rao}^{3}$ \\ ${ }^{1,2}$ Harbin University of Commerce, China \\ ${ }^{3}$ Department of Computer Science and Engineering, Vignan's Institute of Information \\ Technology (A)Visakhapatnam, AP, India \\ 1'chenming4321@126.com ${ }^{3}$ nakkathiru@gmail.com
}

\begin{abstract}
The utilization of deep learning and its related techniques was growing in a faster manner by day to day research. As the technology was developing, the utilization of these technologies also growing in a faster manner. This technology is the latest and advanced technology available in the market today. The utilization of these techniques is providing some good and encouraging results when compared to the previous existing methods and solutions for the current day problems. The implementation of AI, ML and Deep learning methods are providing the researchers some set of new ways and new solutions. These results are encouraging the researchers further such that to go for next level of research by incorporating these techniques in the more and more related areas. Hence, in the current paper, brief details about the deep learning and its applications are presented. The various existing applications and the technologies available in the market can be processed and further can be studied with the other set of applications.
\end{abstract}

Keywords: AI, ML, deep learning, black and white images, security, safety, firewalls, image recognition, hand writing, a translation of data.

\section{Introduction}

Now a day, there is a huge demand and huge famous talks in the society with the researches and in the research community about the developing technologies like artificial intelligence, machine learning and deep learning etc. The utilization of these technologies in the current day research and other future dependent technologies will gives the users with new and mesmerizing output [1]. Deep learning is achieving various extraordinary results by utilizing this technology in various applications. The state of the art technologies are growing day to day by the utilization of these technologies in the current day research. Various problems or big research problems in the society are being given solutions by the utilization of this technology or the other sister technologies like the AI and machine learning. By the utilization of these technologies and other features o thee techniques like AI and ML, several ground level results and output were obtaining in these days. Very few research contributions can be observed in the research societies as this area had started very recently for the utilization and working on the more number of researchers. Now a days, the researchers had started working on these areas

Supported by:

Heilongjiang Provincial Department of Education Technical Research Surface Project（NO.12531149）

Article history:

Received (November 28, 2019), Review Result (February 13, 2019), Accepted (May 2, 2019)

Print ISSN: 2205-8370, elSSN: 2207-5372 IJCSITE

Copyright (C) 2019 GV Press 
with the combination of other research domains and the results were displayed in very few research articles. It is also encourageable for the public to increase the research thoughts on the areas of recent domains with the implementation of these technologies.

The applications of machine learning and its related techniques and algorithms had given the researchers and other research people who were working on these areas, the dynamic results which were very much better than the existing type of results and rules for the further processing of current applications. The users or researchers cannot argue or cannot avoid the set of advantages and other applications that were attaining from using these techniques will be more than the expected ones [2]. When compared with the machine learning techniques, the current deep learning and its related techniques are providing more benefits than the expected ones. In order to protect the data and applications which were running the current day needs to be updated and also to provide such facilities, the user's needs to maintain huge set of computers and servers with huge databases. Providing such huge spaces and other security features to the existing technologies, the user needs to spend more amounts of data on the facilities and their maintenance. Hence, in the current point the deep learning concept is going to play a key role and the researchers attracted towards such advantages only.

Deep learning plays a key role in the points of space allocation and storage of huge databases and maintenance of such huge databases. By using deep learning techniques, the same problem can be resolved by using the storage of data. In deep learning models, the data can be stored in the form of columns in the neural network model. It stores the data in the form of unstructured data. The processing of the data that was stored in neural network models in the deep learning concepts are in the form of human brain structure and imitates the similar functioning of human brains. The decisions will be taken for the availability of data in the form of various predictive reasoning models. As the data is going to be stored in the form of a predictive reasoning model, the data can be processed more easily and can get a good set of results. The results may be dynamic such that to get the updated and new results. This mechanism will provide the capacity such that to handle the huge amount of data and millions of points of data with fast and quickly processing. The data can be processed very quickly and rapidly and always chances will be more to get a good and accurate set of results. The processing of the big systems or the servers will be made easy by the format of this data types and other set of infrastructure for the further processing of huge databases [3].

The mathematical models are being utilized by the deep learning and it's related other models such that to find the solution to the problems which requires mathematical solutions too including the actual output. It is always good to develop a mathematical model such that the results can be actualized with this model and then we can proceed for the actual output and their behaviour with the help of these techniques. For working on various big databases and other sources, current techniques and other related models are required for better working and for better working models such that to get the more good results than the expected ones. For some security reasons, the data can be processed with some advanced models and other predicting technologies and methods such that to avoid the malwares, suspected data and other related issues. The attacks on the networks and other sources also can be verified with the help of these technologies and other related issues.

The security issues and attacks on the networks and other internet which were placed in the companies are increasing from day to day. As a result, the companies and the industries are spending more on these security issues and also acquiring the more and more sophisticated systems from day to day such that to keep their data and other company related data more securely and better services can be provided to the customers. The customers need to get the better services always front he companies such that the data can be provided full security and 
the data of the employees and customers should be kept always a secret and also more secure. In other ways, the important data will be leaked out and the companies will be in big problems. Hence, the companies and industries are going for the usage of the latest trends and technologies like machine learning, AI and deep learning techniques. By using these techniques, the security of these systems can be made more safe and secure. By using these technologies, the security of the data in the companies and other related departments will be controlled and protected by these technologies in better ways [4].

\section{APPLICATIONS OF DEEP LEARNING}

The usage of deep learning and its related techniques and other models can be implemented for working of various equipment. The technology that was established and working on the areas of deep learning today was more updated and cannot be changed for the next two or three years such that users can get better results and the other set of applications. The results that were obtaining from the current set of applications and the techniques will be last for next two years and the researchers can go for the working of these technologies more and more. Some of the important applications, where these deep learning techniques and other related issues were being implemented and tested for the various set of test cases and got good results and the extension of such technologies were always in the process of development. This technology will give us the more and more benefits and the other set of results that can be achieved with the new set of encouraging results. Some of these applications were discussed in detail in the following sections and the details are explained with the help of some diagrams[5]. Set of applications are as follows,

Filling colour to the Black and White images

Addition of sounds and volumes to silent movies,

Translation of the machine and related data automatically

Classification of objects in photos

Handwriting generation and recognition automatically

Image caption generation

Automatic game playing

\subsection{FILLING COLOUR TO BLACK AND WHITE IMAGES}

Now a day, this technique of adding black and white colour to the colour images was growing in demand. The reason was the availability of images with black and white colour were more and always it is interesting for the common public to convert a black and white image to a colour image. The conversion was always a challenging task due to the reason that during conversion proper care should be taken to fill the images with various colours. The filling of colours was very important and the hard task, as the choosing of colour for a particular point of the image or the pixel of the mage was the challenging task. If the correct colour was not chosen for a particular pixel, the meaning of the image or the message of the image might change. The actual understanding of the images will be changed to the other set of meaning by changing or giving the inappropriate colour to the inappropriate location of a pixel of the images [6].

In traditional methods, the mode of colouring or the filling of colour in the black and white images will be done by the hand and human efforts. The human's presence of mind and their talent of filling the colours and the thinking of human beings will be displayed in the images after correction. It is also true that a standard process of filling was not given due to the prospect 
of human beings may change from time to time for filling the images with various colours of data. The time that human being spending on the image for conversion will be from some minutes to hours or in some other way from some hours to some number of days also might take place. Hence, the time will be an independent task here, hence an automated process can be developed here by giving some standard procedures and the other set of processes such that to reduce the processing time and doubt of filling the colours at various locations.

In deep learning, first of all the image will be studied more and more and the details of the image will be observed in detail and then the process of filling the colours in the image pixels will be started. The objects in the images will tried to understand first and then the other objects and human beings in the image will be tried to understand and then followed for further processing. Then, the appropriate colour for the content of the images will be decided such that the colour filling will give a meaningful of image the content and other set of objects in the images. The human approach will be more and accurate for filling of colours.

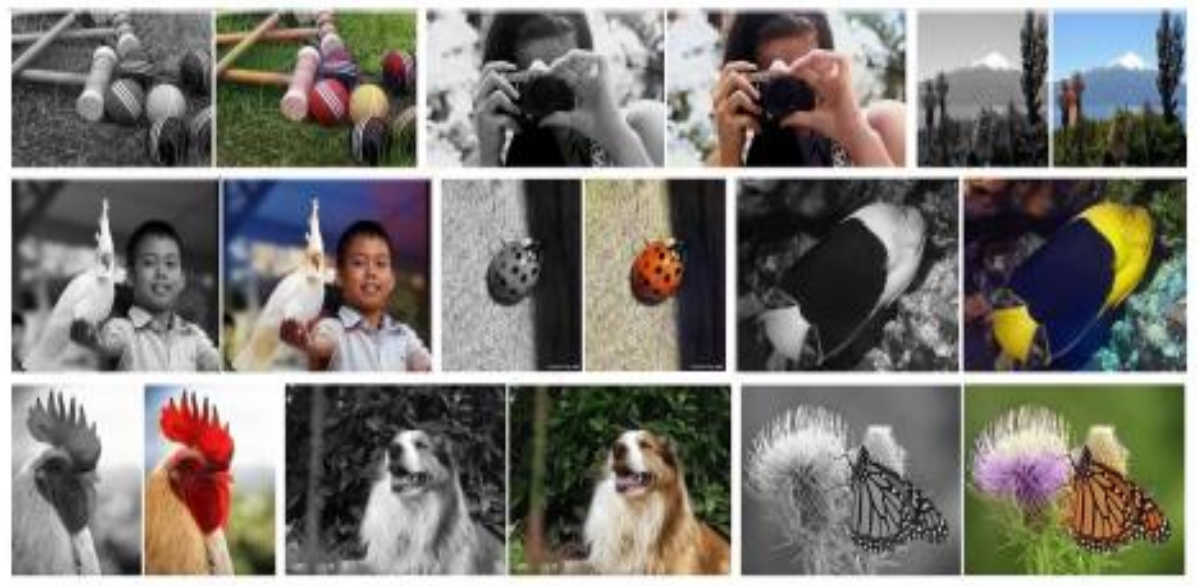

\section{Figure 1. An example for colour filling in black and white images [4]}

The current trend and the other techniques were to fill the colour in the image pixels. This feat was very good and impressive feat that will give the good meaning to the common people. This method or the techniques can be performed by the utilization of the Convolutional neural network model such that to get better outputs. The neural networks will be trained for further processing based on the model ImageNet and other for the filing of the image with good colours. The usage of neural networks and other supervised food layers will give us the good method of finding the good options for filling up of colours.

\subsection{ADDITION OF SOUNDS AND VOLUMES TO SILENT MOVIES}

Now a days, the people started habit of keeping or adding some audio or volume songs to the recorded videos. As the technology for the uploading of videos was growing day to day, the making of videos and uploading of number of videos were increasing a lot. Especially with the growing you tube, this method of making videos and adding some audios to the existing videos had increased and the number of videos in this sense are increasing a lot. In the current scenario, as the YouTube is paying money for the videos with huge likes and dislikes, also the number of views for the videos, the people is more craze about making videos and uploading in the YouTube [7]. It is also used in some situations where some video file is available and audio was not available. In such cases, the video was attached to such video such that to identify the content of the video or in some cases, it is used for the identification of some criminals also. In the current deep learning models, the videos are made in to pieces and the suitable audio can be 
identified based on the previous videos and previous data and the data can be assigned to the videos simply.

The addition of audio to videos was easy but, identifying the suitable song or audio was difficult task. Not only that the fitting of such suitable audio file to the video file was another important task. Hence, some training should be given to the system with the help of the deep learning techniques of artificial neural networks such that the files will be read $n$ number of time. After completing the reading of such files, users are requested to add some more previous files such that the system will try to understand the way of files or type of audio files attached to the type of video files and some classification was done. The classification may be in the form of some sort of videos with some sort of audio files. These audio files are easy to process and easy to understand. Similarly, these audio and video combined videos are easy to process and easy to understand and the size of such videos is very few in data size and can be easily processed and can be easily transferred from one location of the device to some other devices.

\subsection{TRANSLATION OF MACHINE AND RELATED DATA AUTOMATICALLY}

People from various locations from one country are moving to various places in the same country or in some cases, the people are been moving from various locations and coming across various languages. It is always not possible to learn all the languages. Hence, if the researchers will prepare a standard device that can convert any language to the needed language. People travelling from other countries also can use the same device such that they may not have any language communication problem to any other people or users in the society. The technique here follows like given words or phrases in on language will be converted to the other language [7]. The target language is based on the user's choice of interest. For doing such translations, we need to provide the total details about both languages like the input language and the other output language. The database or the knowledge base that was being supplied to the machine will have both languages full data and the rest of the data will be there with rules and whenever there is a need of use, the data can be used.

The conversion of the language or the translation of the language was existing in the society from so many years but the utilization of such deep learning based techniques will give us good results and performance of the machines will be more than the expected ones. The top areas or the applications where these translation techniques are providing good results than the expected ones are the data translation and the image translations. The translation of data is somehow easy than the translation of images. In some cases, it is very hard to understand the content of the images and the content on the images.

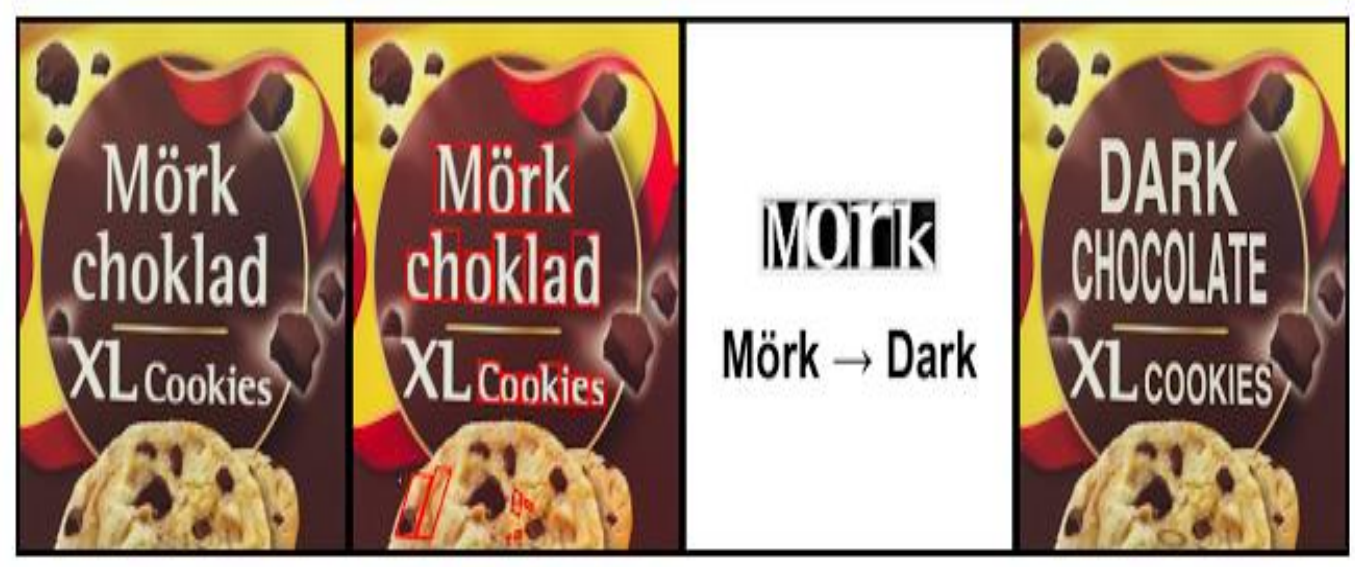




\section{Figure 2. Image translation example for deep learning applications [6]}

The automatic translation of text on the images was some easy to understand in some cases and in some cases it is very hard to even read the data. Always it is not possible to get the clear and good data for the processing, in some cases it may happen that we may not get the full and clear data further processing. Identifying the cont on the images was the first big task and also to identify which language those input data or text was in and to what language we need to convert text. Text translation can be performed without any pre-processing of the sequence, allowing the algorithm to learn the dependencies between words and their mapping to a new language. Stacked networks of large LSTM recurrent neural networks are used to perform this translation.

\subsection{OBJECT CLASSIFICATION AND DETECTION IN PHOTOGRAPHS}

The other important application of deep learning was to identify various data or text and some content on the images. It is very difficult to identify the content on the images and the machine needs to understand the picture first and it has to make the picture in to pixels and tries to identify the type of the object or colour of the objects on the images. In such cases, the colour of the image is also plays a key role. Because, same logic or same method cannot be used for both black and white images and the colour images always. The process should be different. Accordingly, working of the current system or machine will depends on the factors on the images. It is always important to identify the topics or the content $n$ the images. The artificial neural networks can be used for doing such good and big tasks. The networks will be trained with a huge set of previous data and the results will be clearly identified and understood by the machine and such data can be always be in the easy format such that it can be easily imported and exported for other devices for using some other applications.

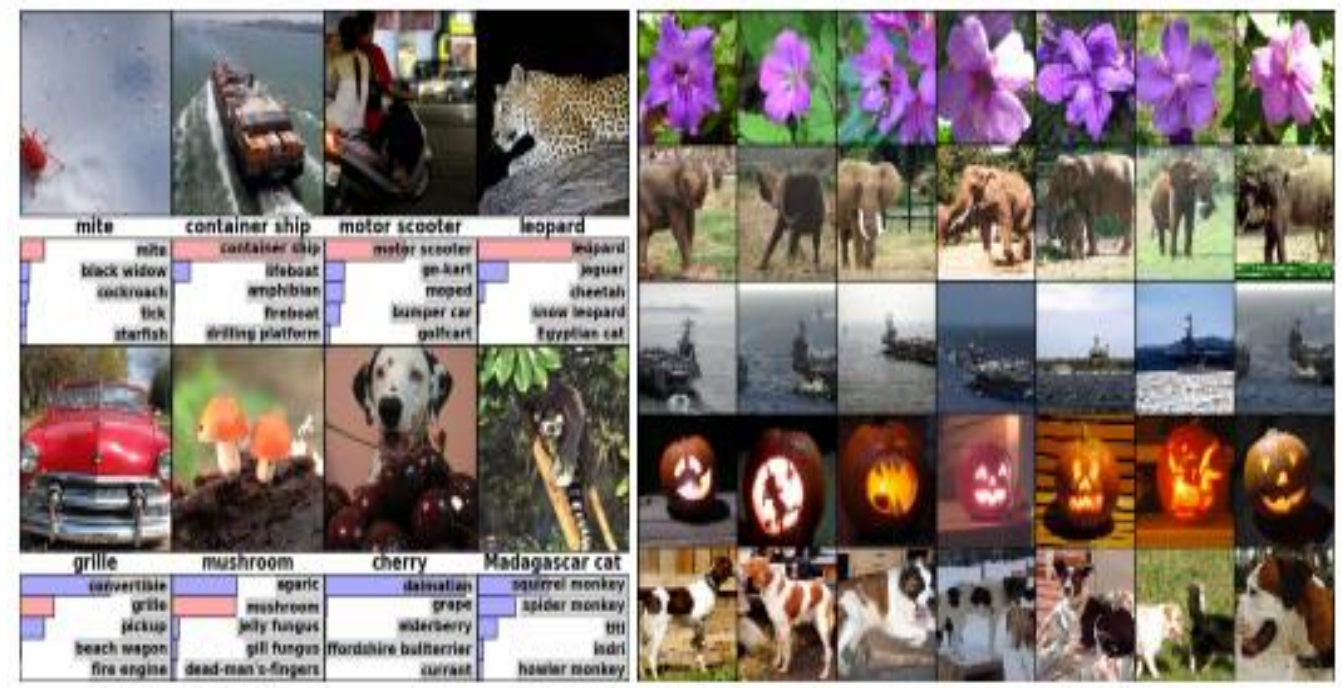

Figure 3. Data Identification on images using Deep Learning techniques

\subsection{AUTOMATIC HANDWRITING GENERATION}

The other important applications of deep learning and its related mechanism were the recognition of hand writing and identifying the written data belongs to a person or not. This sort of applications is 
becoming famous on now a days and it is so useful for several department people in the society. The police can verify the hand writing data of a person whether he given or wrote the same letter or not. With that several criminals can be caught in various cases for different reasons. In the same way, the same application can be used for bank purposes. Already several international and nationalised banks had started this application using. This application helps the clerks in the bank to identify the signature of a customer before performing any financial transactions. The most important and delicate issues are the financial transactions where the signature of the persons or the account holders will play a key role. The identification of person was more important in such cases.

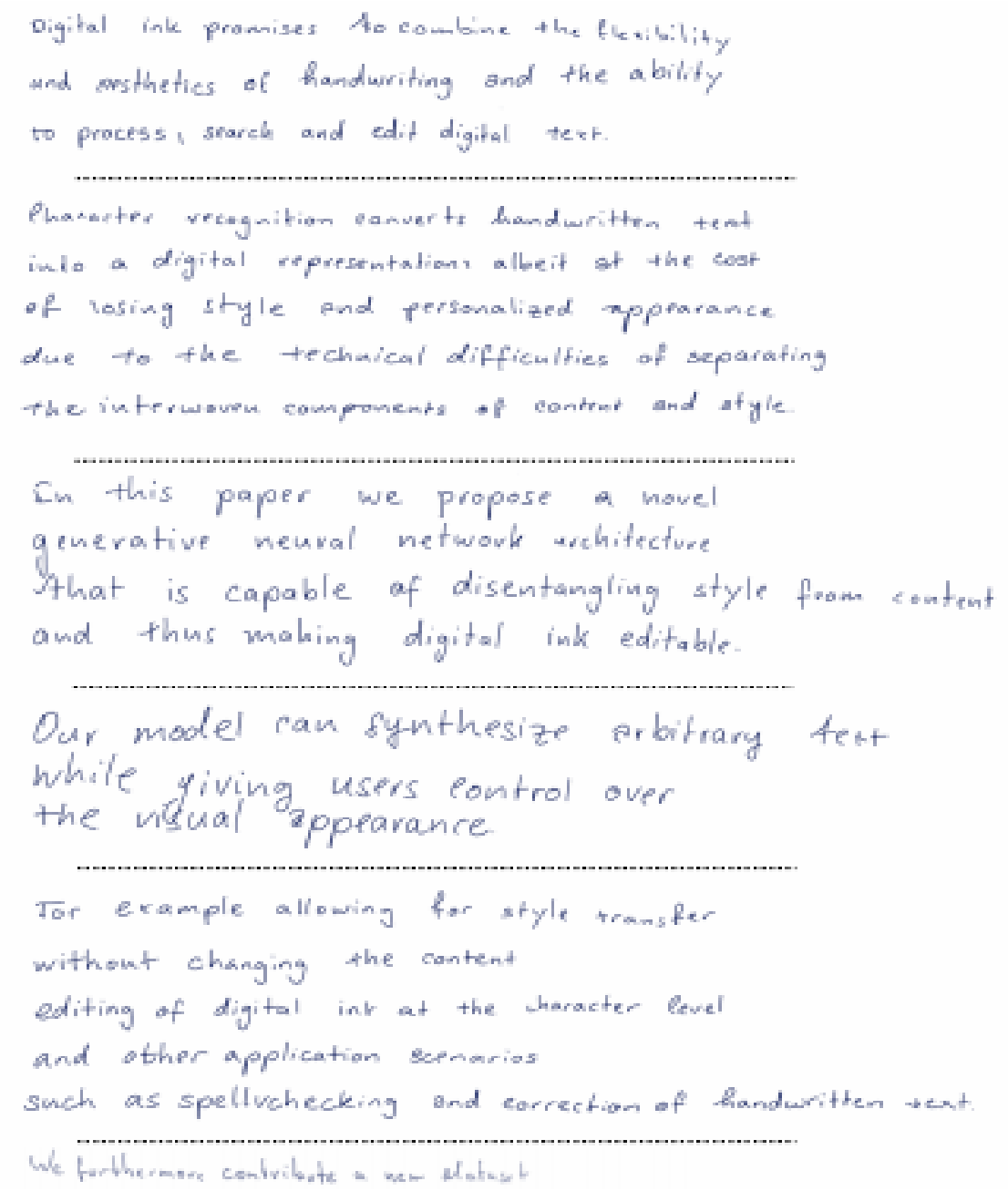

Figure 4. Hand writing recognition example for Deep Learning [3]

If the entire responsibility was given to the employees or the clerks who were working in the banks on such jobs, the people may do some malpractices and also may commit some cheating sort of actions. Hence, by the utilization of these applications with the bank accounts software's, the identification of the account holders signature will be taken care by the software and the rest part will be given to the clerks on the seat. The machines will provide the maximum amount of efficient data and accurate data identifying the signatures of the account holders. The total data of the customers can be stored in the banks databases and whenever it is required, the data can be accessed and can be processed for further applications. 


\section{SOME OTHER APPLICATIONS}

Some of the other applications where the deep learning, AI and ML techniques and other resources were being used for the further development of the technology and other benefits are,

Automatic speech recognition.

Deep Neural Networks for Acoustic Modelling in Speech Recognition.

Automatic speech understanding.

Towards End-to-End Speech Recognition with Recurrent Neural Networks.

Automatically focus attention on objects in images.

Recurrent Models of Visual Attention.

Automatically answer questions about objects in a photograph.

Exploring Models and Data for Image Question Answering.

Automatically turing sketches into photos.

Convolutional Sketch Inversion.

Automatically create stylized images from rough sketches.

Neural Doodle

\section{CONCLUSIONS}

In the current paper, a brief discussion and data about the deep learning and its related applications was presented. A brief idea about various applications and the technologies of deep learning that were being used today in the market was given. The various sub areas of work related with this area of deep learning was also given a light on that. In these days, the utilization of this technology was growing like anything. The applications discussed in the current article may be some but it is not limited too. Some other more applications are also being used this technology. Almost all areas of the research were being taken place on this area and all the researchers started applying this technology and its related topics to their own related areas. By observing and studying the applications that were discussed here will provide a detailed idea on how the applications of deep learning are working and how they can be extended further to other areas of research and also for the extension of other related applications. Hence, all those applications had given in detail in the current paper with some good examples.

\section{References}

[1] Rocio Vargas, Amir Mosavi, Ramon Ruiz, "Deep Learning: A Review”, Advances in Intelligent Systems and Computing, August (2017), Vol.5, No.2, pp.1-10.

[2] https://machinelearningmastery.com/inspirational-applications-deep-learning. [Last accessed on 30-09-(2018)]

[3] Emre Aksan, Fabrizio Pece, Otmar Hiliges, "DeepWriting: Making Digital Ink Editable via Deep Generative Modeling”, Proceedings of the (2018) CHI Conference on Human Factors in Computing Systems, Canada. DOI: $10.1145 / 3173574.3173779$

[4] Richard Zhang, Philip Isola, Alexei A.Efros, "Colorful Image Colorization", European Conference on Computer Vision, Springer, Oct, pp.649-666. (2016), DOI: 10.1007/978-3-319-46487-9_40

[5] Andrej Karpathy, Li Fei-Fei "Deep Visual-Semantic Alignments for Generating Image Descriptions", IEEE Transactions on Pattern Analysis and Machine Intelligence, Vol.39, No.4, pp.664-676. (2015) DOI: 10.1109/TPAMI.2016.2598339

[6] Jason Brownlee, "8 Inspirational Applications of Deep Learning”, A tutorial published on July, 2016. [https://machinelearningmastery.com/inspirational-applications-deep-learning/- Last Accessed on 10-02(2019)] 
[7] Alex Krizhevsky, Ilya Sutskever, Geoffrey E.Hinton "ImageNet classification with deep convolutional neural networks", Communications of the ACM, Jun (2017), Vol.60, No.6, pp.84-90.DOI: 10.1145/3065386 